\title{
Results of the European survey on the assessment of deep molecular response in chronic phase CML patients during tyrosine kinase inhibitor therapy (EUREKA registry)
}

Susanne Möbius ${ }^{1}$, Thomas Schenk ${ }^{1}$, Danny Himsel ${ }^{1}$, Jacqueline Maier ${ }^{2}$, Georg-Nikolaus Franke $^{2}$, Susanne Saussele ${ }^{3}$, Christiane Pott $^{4}$, Hajnalka Andrikovics ${ }^{5}$, Nora Meggyesi ${ }^{5}$, Katerina Machova-Polakova ${ }^{6}$, Hana Zizkova ${ }^{6}$, Tomáš Jurcek ${ }^{7}$, Semir Mesanovic ${ }^{8}$, Renata Zadro ${ }^{9}$, Enrico Gottardi ${ }^{10}$, Jens Haenig ${ }^{11}$, Peter Schuld ${ }^{11}$, Nicholas C. P. Cross $^{12,13}$, Andreas Hochhaus ${ }^{1}$, Thomas Ernst ${ }^{1}$

1 Abteilung Hämatologie/Onkologie, Klinik für Innere Medizin II, Universitätsklinikum Jena, Jena, Germany

2 Abteilung für Hämatologie und internistische Onkologie, Universität Leipzig, Leipzig, Germany

3 III. Medizinische Klinik, Universitätsmedizin Mannheim, Universität Heidelberg, Mannheim, Germany

4 Second Medical Department, University Hospital Schleswig-Holstein, Campus Kiel, Kiel, Germany

5 National Blood Service, Budapest, Hungary; Central Hospital of Southern Pest, Budapest, Hungary

6 Department of Molecular Genetics, Institute of Hematology and Blood Transfusion, Prague, Czech Republic

7 Department of Internal Medicine, Hematology and Oncology, University Hospital Brno and Faculty of Medicine, Masaryk University Brno, Brno, Czech Republic

8 Pathology Department, University Clinical Center Tuzla, Tuzla, Bosnia and Herzegovina

9 University Hospital Center Zagreb and University of Zagreb Faculty of Pharmacy and Biochemistry, Zagreb, Croatia

10 Division of Internal Medicine \& Hematology, University of Turin, Orbassano, Italy

11 Novartis Oncology, Global Medical Affairs, Basel, Switzerland 
12 Wessex Regional Genetics Laboratory, Salisbury NHS Foundation Trust, Salisbury, UK

13 Faculty of Medicine, University of Southampton, Southampton, UK

Corresponding author:

PD Dr. Thomas Ernst, Abteilung Hämatologie und Internistische Onkologie, Klinik für Innere Medizin II, Universitätsklinikum Jena, Am Klinikum 1, 07740 Jena, Germany. Tel. +49 $36419324201 ;$ Fax: +49 36419324202

Email: thomas.ernst@med.uni-jena.de 


\section{Abstract}

Purpose: The advent of tyrosine kinase inhibitor (TKI) therapies has revolutionized the treatment of chronic myeloid leukemia (CML). The European LeukemiaNet (ELN) recommends quantification of BCR-ABL1 transcripts by real-time quantitative PCR every three months during TKI treatment. Since a proportion of patients in deep molecular response (DMR: $\mathrm{MR}^{4}, \mathrm{MR}^{4.5}, \mathrm{MR}^{5}$ ) maintain remission after treatment stop, assessment of DMR is crucial. However, systematically collected molecular data, monitored with sensitive standardized assays, are not available outside clinical trials.

Methods: Data were collected on the standardized assessment of molecular response in the context of real life practice. BCR-ABL1 transcript levels after $>2$ years of TKI therapy were evaluated for DMR. Since standardized molecular monitoring is a prerequisite for treatment discontinuation, central surveillance of the performance of the participating laboratories was carried out.

Results: Between 2014-2017, 3,377 peripheral blood samples from 1,117 CML patients were shipped to 11 standardized reference laboratories in six European countries. BCRABL1 transcript types were b3a2 (41.63\%), b2a2 (29.99\%), b2a2/b3a2 (3.58\%) and atypical $(0.54 \%)$. For $23.72 \%$ of patients, the initial transcript type had not been reported. Response levels (EUTOS laboratory) were: no MMR, $n=197$ (6.51\%); MMR, $\mathrm{n}=496$ (16.40\%); MR ${ }^{4}, \mathrm{n}=685$ (22.64\%); $\mathrm{MR}^{4.5}, \mathrm{n}=937$ (30.98\%); $\mathrm{MR}^{5}, \mathrm{n}=710$ (23.47\%). With a Cohen's kappa coefficient of 0.708 , a substantial agreement between EUTOScertified and local laboratories was shown.

Conclusions: Multicenter DMR assessment is feasible in the context of real life clinical practice in Europe. Information on the BCR-ABL1 transcript type at diagnosis is crucial to accurately monitor patients' molecular response during or after TKI therapy. 
Keywords: chronic myeloid leukemia, CML, BCR-ABL, molecular monitoring, deep molecular remission, treatment free remission, TFR, standardization, Eureka

\section{Introduction}

With the advent of the tyrosine kinase inhibitor (TKI) imatinib in 2001 treatment of CML changed fundamentally, the disease became well treatable with the majority of patients reaching stable deep molecular response (DMR: $\mathrm{MR}^{4}, \mathrm{MR}^{4.5}, \mathrm{MR}^{5}$ ). Nevertheless, since cure is not possible at this time, lifelong molecular monitoring must be ensured for an increasing number of patients. During the last years different milestones were established that have been shown to be of predictive value. Patients with complete cytogenetic remission (CCyR) have a life expectancy similar to the general population (Gambacorti-Passerini et al. 2011). Furthermore, reaching CCyR after 6 months of therapy represents an important prognostic factor for progression-free survival (Hochhaus et al. 2009). An important milestone in CML therapy is the achievement of a major molecular response (MMR), which has been shown to be associated with lower relapse rates and therefore higher progression-free survival (Hughes et al. 2003). Furthermore, higher survival rates could be observed for patients who reached MMR after 12 or 18 month of imatinib therapy, underlining the importance of early responses in combination with low risk scores (Sokal, Euro or EUTOS long term survival [ELTS] scores) as prognostic markers for long-term outcome (Hochhaus et al. 2017). These findings were adopted by the European LeukemiaNet (ELN) recommendations for the management of CML. According to current ELN recommendations (Baccarani et al. 2015; Baccarani et al. 2013), achieving a major molecular response (MMR) following 12 months TKI treatment is considered an optimal response to therapy. With the advent of $2^{\text {nd }}$ generation TKI more and more patients achieved progressively deeper responses (Kantarjian et al. 2010; Saglio et al. 2010). Furthermore, lower progression rates, faster responses as well as higher rates of MMR were observed with $2^{\text {nd }}$ generation TKI (Jabbour et al. 2014; Kantarjian et al. 2011; Saglio et al. 2010). 
With the increasing proportion of patients achieving a DMR the question was raised, whether lifelong TKI administration is necessary for all patients or if TKI discontinuation may be an option in some cases (Hehlmann et al. 2014). In the German CML IV study, the achievement of $\mathrm{MR}^{4.5}$ was identified as a molecular predictor of long-term outcome and may therefore represent an important milestone if treatment discontinuation is considered (Hehlmann et al. 2014). In addition the duration of DMR seems to play a crucial role (Mahon et al. 2010; Saussele et al. 2016). Results from the EURO-SKI trail suggest, that DMR duration represents the most important predictive factor for remaining in treatment free remission (TFR) at 6 month after treatment discontinuation (Saussele et al. 2018). Since the majority of patients will reach DMR after several years of TKI therapy(Hehlmann et al. 2014; Kalmanti et al. 2015), the proportion of samples with very low BCR-ABL1 levels will further increase in the next years and therefore the number of patients eligible for TFR. Different studies investigating TKI cessation showed, that 40$50 \%$ of the patients remain in deep molecular remission, while more than half of the patients will suffer a molecular relapse characterized as MMR loss (Mahon et al. 2010; Ross et al. 2013; Saussele et al. 2016). Nevertheless, as of yet there is no method to identify patients who can successfully cease therapy. A fundamental requirement to find such a method is the reliable, reproducible and comparable molecular monitoring of patients.

A major step in the standardization process was the harmonization of the nomenclature and definition of response levels: molecular results are expressed on the international scale (IS) as $\log _{10}$ reduction from a standardized baseline, defined in the course of the International Randomized Study of Interferon and STI571 (IRIS) (Hughes et al. 2006; Hughes et al. 2003). To ensure comparability of results between laboratories and to compensate for deviations due to technical differences, the use of different assay formats or components, for example for RNA isolation or reverse transcription, laboratory-specific conversion factors were introduced (Branford et al. 2006; Branford et al. 2008; Muller et al. 2009).

Recently, the European Treatment and Outcome Study (EUTOS) for CML improved the standardization throughout Europe to ensure quality controlled, standardized molecular monitoring. The EUTOS collaboration provides more than 50 reference laboratories 
Europe-wide with validated control samples as well as recommendations, standardized procedures and protocols for BCR-ABL1 transcript quantification (Cross et al. 2015a; Gabert et al. 2003; Hughes et al. 2006). Moreover new technologies for molecular monitoring have been taken into account to improve sensitivity and specificity of BCRABL1 measurements in the clinical routine. For further simplification of the standardization process, reference panels were designed recently to enable a more effective and efficient IS calibration also accessible for smaller laboratories without the need for a reference laboratory (Cross et al. 2016; White et al. 2015).

Dependable and comparable quantification of BCR-ABL1 transcript levels are crucially important to make timely important treatment decisions and will assist to define the parameters for treatment discontinuation (Baccarani et al. 2013; Saussele et al. 2016). To evaluate the accessibility of standardized DMR measurements in Europe the EUREKA registry as part of EUTOS launched in 2014. The purpose of this laboratory registry was the collection of data on the standardized assessment of molecular response in the context of real life clinical practice in European countries. BCR-ABL1 transcript levels after at least two years of TKI therapy were evaluated for the occurrence of DMR rates, to determine, to what extent DMR assessment is implemented outside of clinical trials.

The objectives of the EUREKA registry were: (i) to determine the proportion of CML patients in DMR $\left(\mathrm{MR}^{4}, \mathrm{MR}^{4.5}, \mathrm{MR}^{5}\right)$; (ii) to demonstrate feasibility and accuracy of deep molecular response analysis in context of real life clinical practice in different countries in Europe, and (iii) to compare the response level obtained in the local laboratory with the response level reported by the standardized (EUTOS) laboratory.

\section{Methods}

The EUREKA registry was designed as a non-interventional laboratory registry and did not impose a therapy protocol, a diagnostic or therapeutic procedure or a visit schedule. Patients were treated according to routine medical practice and only these data were 
collected as part of the registry. The eleven participating laboratories must have passed the EUTOS certification for deep molecular response assessment $\left(\mathrm{MR}^{4.5}\right)$. Adult chronic phase BCR-ABL1 positive CML patients under treatment with TKIs at any prescribed dose were included. The patients should have received TKI treatment for a minimum of 2 years at registry entry. After this time of therapy a high proportion of patients is expected to have reached stable DMR. Written informed consent was obtained before enrolling patients. EDTA blood samples $(20 \mathrm{ml})$ were sent once or repeatedly from the same individual with at least 10 weeks interval between samples along with anonymized information of the patients $\mathrm{CML}$ history from the referring physician to the participating EUTOS-certificated laboratory. At the time of registration anonymized information were collected on patient demographics, date of CML diagnosis, type of BCR-ABL1 transcript, initial prognostic score (EUTOS and Sokal scores), past and current treatments as well as current molecular response according to the local laboratory. Data were collected via a multicenter web based data registry-portal (OpenClinica database). A Cohen's kappatest was performed to determine the agreement between the result from the EUTOScertificated laboratory and the most recent local measurement (Landis and Koch 1977). In all participating laboratories ABL1 was used as reference gene.

\section{Results}

Eleven laboratories in six European countries (Germany, Bosnia and Herzegovina, Hungary, Czech Republic, Croatia and Italy) participated (Table 1). Between 2014 and $2017,3,377$ samples of 1,117 patients were analyzed in total. In total 1,534 samples from female patients and 1,843 samples from males have been registered in the database. The median age of this patient cohort is 61 years (range, 19-90 years). The EUTOS score was reported for 747 patients. The EUTOS score was low for 661 patients and high for 86 patients. The overall distribution of the current TKIs and therapy lines at the time of sample analysis is shown in Tables 2 and 3. Imatinib (84.3\%) was the most common TKI for first line therapy followed by nilotinib (13\%). For second line therapy 
nilotinib was most frequently used (58.6\%) followed by dasatinib $(23.3 \%)$ and imatinib (16.9\%). Dasatinib (42.9\%) and nilotinib (34.5\%) were mostly prescribed if a third line therapy was needed. The distribution of the BCR-ABL1 transcript types of the analyzed samples is shown in Table 4. The initial transcript type was reported as unknown by the referring physician for $24.4 \%$ of patients $(n=285)$, for $0.53 \%(n=6)$ it was only known that a typical transcript (b3a2 or b2a2) is present.

EUTOS laboratories were able to confirm a deep molecular response in $76.0 \%$ of the RQ-PCR analyses. The participating EUTOS-certificated laboratories demonstrated a sufficient measuring quality of the internal reference gene ABL1 according to the current guidelines: $99 \%$ of the analyzed samples showed ABL1 transcript numbers $>10,000$, therefore enabling the scoring of at least $\mathrm{MR}^{4}$ (Cross et al. 2015b). Figure 1 shows the comparison between molecular remission evaluation of the most recent local measurement and the measurement obtained in the standardized EUTOS laboratories. Of 3,377 analyzed samples in total, 3,025 were applicable for the Cohen's kappa-test, for the remaining 352 samples only the result from the EUTOS-certified laboratory, but not from the local laboratory was available. The Cohen's kappa-test showed a substantial agreement with a Cohen's kappa $(\mathrm{K})$ coefficient of 0.708 .

The analysis of the registry data highlighted a difference regarding the ability to reach DMR between patients with continuous first line therapy and patients with a TKI switch. $85.2 \%$ of the samples of patients with ongoing first line TKI therapy reached a DMR. In contrast, only $67.6 \%$ of the samples of patients, who had to change TKI therapy due to resistance or intolerance before, reached a DMR. This analysis was based on the results obtained in the standardized laboratory for 2,960 of 3,377 samples. For the remaining 417 samples no complete datasets, for example regarding therapy information, were available.

\section{Discussion}


Molecular monitoring in CML must be ensured over long periods of time with the number of CML patients continuously increasing (Huang et al. 2012). A prerequisite for future recommendations for patients with durable DMR and the possibility to reach a treatment free remission is the unrestricted availability of a continuous, reliable and comparable BCR-ABL1 monitoring. The present study evaluated how well DMR assessment has been implemented thus far in the context of real life clinical practice in 6 European countries. By analyzing a large amount of samples, the EUREKA registry demonstrates the accessibility to a reliable BCR-ABL1 monitoring for CML patients with deep molecular responses outside of clinical studies. We could demonstrate a substantial agreement between the most recent local measurement and the results within the standardized EUTOS laboratory. Therefore patients from the participating countries are eligible for clinical studies relying on the values obtained in the respective local laboratories. A major drawback in the study design was that not identical, but consecutive samples were compared between the local and standardized laboratory. Even if the samples were collected in only short time intervals, changes in the BCRABL1 transcript level caused by improved or worsened response to therapy are possible. Small variations of the molecular results may be caused by RQ-PCR measurement deviations of the different assays, but also by real differences of BCRABL1 transcript levels. Furthermore local laboratories did not exist at all locations. Therefore in some cases the local and standardized laboratory was identical. Nevertheless, this emphasizes the good availability of dependable, standardized molecular monitoring at the respective locations, underlining that there is no disadvantage for patients who are not included in clinical studies.

The high percentage of samples with unknown BCR-ABL1 transcript types (24.4\%) is a major concern and underlines the need for strict documentation of the transcript type at the time of CML diagnosis. Since the standardized assays only target the typical BCRABL1 transcripts b2a2 (e14a2) and b3a2 (e13a2), in patients with unknown BCR-ABL1 transcript types false-negative results cannot be excluded as approximately 2-3\% of CML patients show atypical transcript variants (Foroni et al. 2011). For such cases, specific bespoke assays that target the individual fusion subtype are necessary. Especially when treatment discontinuation is considered, an undetected atypical 
transcript may have fatal consequences. Additionally in the present study, the agreement may be overestimated due to unknown atypical transcript types leading to falsely undetectable disease in both measurements. To overcome this problem, a central register for CML patients would be desirable. Thus far, recommendations for treatment discontinuation can be given only for patients with the typical transcript types (Foroni et al. 2011).

Furthermore, the EUREKA registry showed that the term "CMR" (complete molecular response) is still in use in some local laboratories. Since the sensitivity of the assay is not clearly described by terms like "CMR" or "UMRD" (undetectable minimal residual disease), the actual depth of response is unknown in these molecular response reports and should therefore no longer be used for CML monitoring (Cross et al. 2012; Saussele et al. 2016). Particularly, when treatment discontinuation is considered, the use of a uniform nomenclature, that ensures sensitivity by defined numbers of the reference gene is essential (Cross et al. 2015b; Cross et al. 2012). Otherwise the response to therapy may be judged too favorable in some cases, creating inadequate conditions prior to stop of treatment on the one hand and the risk of an unrecognized BCR-ABL1 increase during TFR on the other hand. Even patients who maintained TFR still showed low level BCR-ABL1 in most cases, as the malignant cell clones seem not to be fully eradicated (Mahon 2016; Ross et al. 2013). Even after successful discontinuation, it is not sure yet, if and when molecular monitoring can be stopped. Since monitoring should be performed more often during the first months of TFR (Cross et al. 2018) the increasing numbers of patients in TFR that can be expected in the next years will increase the workload for laboratories.

In conclusion our registry has shown that CML patients in 6 European countries have access to reliable molecular monitoring even outside clinical trials. For a majority of patients the assessment of DMR is ensured also in non-standardized laboratories, which forms the basis for the establishment of criteria that permits safe discontinuation of TKI therapy. 


\section{Compliance with ethical standards}

Funding: This study was funded by Novartis Oncology within the European Treatment and Outcome Study (EUTOS).

Conflict of interest: All authors declare that they have no conflict of interest.

Ethical approval: All procedures performed in studies involving human participants were in accordance with the ethical standards of the institutional and/or national research committee and with the 1964 Helsinki declaration and its later amendments or comparable ethical standards. The study has been approved by the ethics committee of the Jena University Hospital, Jena, Germany (No. 3944-12/12).

Informed consent Informed consent was obtained from all individual participants included in the study.

\section{References}

Baccarani M, Castagnetti F, Gugliotta G, Rosti G (2015) A review of the European LeukemiaNet recommendations for the management of CML Annals of hematology 94 Suppl 2:S141-147 doi:10.1007/s00277-015-2322-2

Baccarani $\mathrm{M}$ et al. (2013) European LeukemiaNet recommendations for the management of chronic myeloid leukemia: 2013 Blood 122:872-884 doi:10.1182/blood-2013-05-501569

Branford S et al. (2006) Rationale for the recommendations for harmonizing current methodology for detecting BCR-ABL transcripts in patients with chronic myeloid leukaemia Leukemia 20:1925-1930 doi:10.1038/sj.leu.2404388

Branford S et al. (2008) Desirable performance characteristics for BCR-ABL measurement on an international reporting scale to allow consistent interpretation of individual patient response and comparison of response rates between clinical trials Blood 112:3330-3338 doi:10.1182/blood-2008-04-150680

Cross NC, Hochhaus A, Muller MC (2015a) Molecular monitoring of chronic myeloid leukemia: principles and interlaboratory standardization Annals of hematology 94 Suppl 2:S219-225 doi:10.1007/s00277-015-2315-1

Cross NC et al. (2015b) Laboratory recommendations for scoring deep molecular responses following treatment for chronic myeloid leukemia Leukemia 29:9991003 doi:10.1038/leu.2015.29 
Cross NC et al. (2016) Development and evaluation of a secondary reference panel for BCR-ABL1 quantification on the International Scale Leukemia 30:1844-1852 doi:10.1038/leu.2016.90

Cross NC, White HE, Muller MC, Saglio G, Hochhaus A (2012) Standardized definitions of molecular response in chronic myeloid leukemia Leukemia 26:2172-2175 doi:10.1038/leu.2012.104

Cross NCP et al. (2018) Consensus on BCR-ABL1 reporting in chronic myeloid leukaemia in the UK British journal of haematology 182:777-788 doi:10.1111/bjh.15542

Foroni $L$ et al. (2011) Guidelines for the measurement of BCR-ABL1 transcripts in chronic myeloid leukaemia British journal of haematology 153:179-190 doi:10.1111/j.1365-2141.2011.08603.x

Gabert J et al. (2003) Standardization and quality control studies of 'real-time' quantitative reverse transcriptase polymerase chain reaction of fusion gene transcripts for residual disease detection in leukemia - a Europe Against Cancer program Leukemia 17:2318-2357 doi:10.1038/sj.leu.2403135

Gambacorti-Passerini C et al. (2011) Multicenter independent assessment of outcomes in chronic myeloid leukemia patients treated with imatinib Journal of the National Cancer Institute 103:553-561 doi:10.1093/jnci/djr060

Hehlmann R et al. (2014) Deep molecular response is reached by the majority of patients treated with imatinib, predicts survival, and is achieved more quickly by optimized high-dose imatinib: results from the randomized CML-study IV Journal of clinical oncology : official journal of the American Society of Clinical Oncology 32:415-423 doi:10.1200/JCO.2013.49.9020

Hochhaus A et al. (2017) Long-Term Outcomes of Imatinib Treatment for Chronic Myeloid Leukemia The New England journal of medicine 376:917-927 doi:10.1056/NEJMoa1609324

Hochhaus A et al. (2009) Six-year follow-up of patients receiving imatinib for the first-line treatment of chronic myeloid leukemia Leukemia 23:1054-1061 doi:10.1038/leu.2009.38

Huang X, Cortes J, Kantarjian H (2012) Estimations of the increasing prevalence and plateau prevalence of chronic myeloid leukemia in the era of tyrosine kinase inhibitor therapy Cancer 118:3123-3127 doi:10.1002/cncr.26679

Hughes T et al. (2006) Monitoring CML patients responding to treatment with tyrosine kinase inhibitors: review and recommendations for harmonizing current methodology for detecting BCR-ABL transcripts and kinase domain mutations and for expressing results Blood 108:28-37 doi:10.1182/blood-2006-01-0092

Hughes TP et al. (2003) Frequency of major molecular responses to imatinib or interferon alfa plus cytarabine in newly diagnosed chronic myeloid leukemia The New England journal of medicine 349:1423-1432 doi:10.1056/NEJMoa030513

Jabbour E et al. (2014) Early response with dasatinib or imatinib in chronic myeloid leukemia: 3-year follow-up from a randomized phase 3 trial (DASISION) Blood 123:494-500 doi:10.1182/blood-2013-06-511592

Kalmanti L et al. (2015) Safety and efficacy of imatinib in CML over a period of 10 years: data from the randomized CML-study IV Leukemia 29:1123-1132 doi:10.1038/leu.2015.36 
Kantarjian $\mathrm{H}$ et al. (2010) Dasatinib versus imatinib in newly diagnosed chronic-phase chronic myeloid leukemia The New England journal of medicine 362:2260-2270 doi:10.1056/NEJMoa1002315

Kantarjian HM et al. (2011) Nilotinib versus imatinib for the treatment of patients with newly diagnosed chronic phase, Philadelphia chromosome-positive, chronic myeloid leukaemia: 24-month minimum follow-up of the phase 3 randomised ENESTnd trial The Lancet Oncology 12:841-851 doi:10.1016/s14702045(11)70201-7

Landis JR, Koch GG (1977) The measurement of observer agreement for categorical data Biometrics 33:159-174

Mahon FX (2016) Discontinuation of TKI therapy and 'functional' cure for CML Best practice \& research Clinical haematology 29:308-313 doi:10.1016/j.beha.2016.10.014

Mahon FX et al. (2010) Discontinuation of imatinib in patients with chronic myeloid leukaemia who have maintained complete molecular remission for at least 2 years: the prospective, multicentre Stop Imatinib (STIM) trial The Lancet Oncology 11:1029-1035 doi:10.1016/s1470-2045(10)70233-3

Muller MC et al. (2009) Harmonization of molecular monitoring of CML therapy in Europe Leukemia 23:1957-1963 doi:10.1038/leu.2009.168

Ross DM et al. (2013) Safety and efficacy of imatinib cessation for CML patients with stable undetectable minimal residual disease: results from the TWISTER study Blood 122:515-522 doi:10.1182/blood-2013-02-483750

Saglio $\mathrm{G}$ et al. (2010) Nilotinib versus imatinib for newly diagnosed chronic myeloid leukemia The New England journal of medicine 362:2251-2259 doi:10.1056/NEJMoa0912614

Saussele S et al. (2018) Discontinuation of tyrosine kinase inhibitor therapy in chronic myeloid leukaemia (EURO-SKI): a prespecified interim analysis of a prospective, multicentre, non-randomised, trial The Lancet Oncology 19:747-757 doi:10.1016/S1470-2045(18)30192-X

Saussele S, Richter J, Hochhaus A, Mahon FX (2016) The concept of treatment-free remission in chronic myeloid leukemia Leukemia 30:1638-1647 doi:10.1038/leu.2016.115

White $\mathrm{H}$ et al. (2015) A certified plasmid reference material for the standardisation of BCR-ABL1 mRNA quantification by real-time quantitative PCR Leukemia 29:369376 doi:10.1038/leu.2014.217 


\section{Figure legend}

Figure 1 Response levels of 3,025 samples reported by the local laboratories in comparison to the standardized EUTOS laboratories. The majority of samples were evaluated correctly. Dark green: full accordance; green: accordance; yellow: discrepancy; red: major discrepancy. 


\section{Tables}

Table 1: Number of samples and patients in the EUREKA registry.

\begin{tabular}{|l|c|c|}
\hline $\begin{array}{l}\text { Country (central } \\
\text { laboratory) }\end{array}$ & Samples (n) & Patients (n) \\
\hline $\begin{array}{l}\text { Germany } \\
\text { (Jena, Mannheim, } \\
\text { Leipzig, Kiel) }\end{array}$ & 1408 & 303 \\
\hline $\begin{array}{l}\text { Bosnia and } \\
\text { Herzegovina } \\
\text { (Tuzla) }\end{array}$ & 657 & 70 \\
\hline $\begin{array}{l}\text { Hungary } \\
\text { (Budapest) }\end{array}$ & 536 & 394 \\
\hline $\begin{array}{l}\text { Czech Republic } \\
\text { (Prague, Brno) }\end{array}$ & 393 & 221 \\
\hline $\begin{array}{l}\text { Croatia } \\
\text { (Zagreb) }\end{array}$ & 340 & $\mathbf{1 1 1 7}$ \\
\hline $\begin{array}{l}\text { Italy } \\
\text { (Turin) }\end{array}$ & $\mathbf{3 3 7 7}$ & 43 \\
\hline Total & 43 & \\
\hline
\end{tabular}

Table 2: Current TKI at time of sample analysis.

\begin{tabular}{|c|c|c|c|c|c|}
\hline Imatinib & Nilotinib & Dasatinib & Ponatinib & Bosutinib & Unknown \\
\hline $\mathrm{N}=1771$ & $\mathrm{~N}=985$ & $\mathrm{~N}=351$ & $\mathrm{~N}=20$ & $\mathrm{~N}=14$ & $\mathrm{~N}=543$ \\
\hline $54.7 \%$ & $30.0 \%$ & $10.8 \%$ & $0.6 \%$ & $0.4 \%$ & $16.8 \%$ \\
\hline
\end{tabular}


Table 3: Treatment line at the time of sample analysis.

\begin{tabular}{|c|c|c|}
\hline First line & Second line & Third line \\
\hline $\mathrm{N}=1791$ & $\mathrm{~N}=1185$ & $\mathrm{~N}=310$ \\
\hline $54.5 \%$ & $36.1 \%$ & $9.4 \%$ \\
\hline
\end{tabular}

Table 4: Type of BCR-ABL transcripts of the analyzed samples

\begin{tabular}{|l|l|l|}
\hline Transcript & Patients (n) & $\%$ \\
\hline b2a2 (e13a2) & 335 & 29.99 \\
\hline b3a2 (e14a2) & 465 & 44.63 \\
\hline b3a3 (e14a3) & 1 & 0.09 \\
\hline $\begin{array}{l}\text { b2a2 + b3a2 } \\
\text { (e13a2 + e14a2) }\end{array}$ & 40 & 3.58 \\
\hline e19a2 & 2 & 0.18 \\
\hline e1a2 & 2 & 0.18 \\
\hline e6a2 & 1 & 0.09 \\
\hline Unknown & 265 & 23.72 \\
\hline Total & 1117 & \\
\hline
\end{tabular}


Figure 1

\begin{tabular}{|c|c|c|c|c|c|}
\hline No MMR & 1 & 3 & 7 & 36 & 158 \\
\hline MMR & 13 & 28 & 98 & 349 & 28 \\
\hline $\mathrm{MR}^{4}$ & 73 & 176 & 420 & 83 & 2 \\
\hline $\mathrm{MR}^{4.5}$ & 190 & 583 & 125 & 25 & 5 \\
\hline $\mathrm{MR}^{5}$ & 394 & 138 & 31 & 3 & 2 \\
\hline “CMR" & 39 & 9 & 4 & 0 & 2 \\
\hline & $\mathrm{MR}^{5}$ & $\mathrm{MR}^{4.5}$ & $\mathrm{MR}^{4}$ & MMR & No MMR \\
\hline & & dizec & DS labor & & \\
\hline
\end{tabular}

